\title{
Altered Sr Atomic Environment in $\mathrm{La}_{2-x} \mathrm{Sr}_{x} \mathrm{CuO}_{4}$
}

\author{
D. Haskel and E.A. Stern
}

Department of Physics, Box 351560, University of Washington, Seattle, WA 98195, U.S.A.

\begin{abstract}
The $\mathrm{Sr}$ local atomic environment in the high $\mathrm{T}_{c}$ superconductor $\mathrm{La}_{2-x} \mathrm{Sr}_{x} \mathrm{CuO}_{4}$ was probed by polarized XAFS measurements at the $\mathrm{Sr} K$-edge on oriented powder samples with $x=0.075,0.1$ at $\mathrm{T}=20 \mathrm{~K}$. We confirmed that $\mathrm{Sr}$ indeed substitutes at the La site in this compound, but alters the first neighbor oxygen environment. This was found by determining the location of the neighboring metal atoms up to the fifth shell distances through fitting the $\mathrm{Sr}$ XAFS, and comparing with La $K$-edge polarized XAFS in the same samples and with diffraction results. The significant distortions in the immediate oxygen environment of the $\mathrm{Sr}$ atoms can be an important factor contributing to the mechanism of the structural and high $T_{c}$ phase transitions in these cuprates.
\end{abstract}

New evidence for the presence of $\mathrm{Sr}$-induced distortions in the $\mathrm{La}_{2-x} \mathrm{Sr}_{x} \mathrm{CuO}_{4}$ system comes from recent X-ray diffuse scattering experiments[1] where strong diffuse scattered intensity was observed in $c^{*}$-cuts of reciprocal space in the neighborhood of bragg peaks. The momentum transfer dependence of the measured intensity implied distortions with a remarkable $c$-axis rms displacement $\left\langle u_{z}^{2}\right\rangle^{1 / 2} \simeq 0.4 \AA$. Sr $K$-edge XANES results[2] were previously interpreted to conclude that the introduction of $\mathrm{Sr}$ under normal preparation conditions leads to the removal of the $\mathrm{O}(2)$ apical oxygen to the Sr and the creation of an oxygen defect in a nearby interstitial site. Recent XANES theoretical calculations[3], however, showed that the XANES features attributed by Tan et. al to the removal of the apical oxygen to the Sr can be reproduced with the same oxygen configuration as about the La atoms. This controversy exemplifies the need of a quantitative approach to the question of Sr-induced structural distortions in $\mathrm{La}_{2-x} \mathrm{Sr}_{x} \mathrm{CuO}_{4}$.

In this work, the $\mathrm{Sr}$ and La atomic environments were studied separately by tuning the X-ray energy to match the corresponding inner-shell electron excitation of either atom. The orientation dependence of the XAFS signal was exploited by rotating the $c$-axis magnetically-aligned-powder relative to the linearly polarized electric field vector of the synchrotron radiation. The high sensitivity of nearly collinear multiple scattering (MS) paths to the presence of the intervening focusing atom was used to determine the occupancy of the $O(2)$ apical oxygen to the Sr atoms.

Sr $K$-edge measurements were performed in transmission at beamline X-11A of the National Synchrotron Light Source, using a $\mathrm{Si}(111)$ double-crystal monochromator. Though aligned along the $c$-axis, the powder was randomly oriented in the $a b$-plane. For such a case, the XAFS of a single scattering (SS) path $i$ at an angle $\theta_{i}$ with the $c$-axis is

$$
\chi_{c}^{(i)}(k)=3 \cos ^{2} \theta_{i} \chi_{r a n}^{(i)}(k) ; \chi_{a b}^{(i)}(k)=3 \frac{\sin ^{2} \theta_{i}}{2} \chi_{r a n}^{(i)}(k),
$$

with $\chi_{c}(k), \chi_{a b}(k)$ being the measured XAFS with the electric field parallel to the $c$ and $a b$ orientations, respectively, and $\chi_{\text {ran }}(k)$ the XAFS signal of an unoriented powder. We used FEFF 6 as our theoretical standard[4] to calculate the random XAFS $\chi_{r a n}(k)$ using the average atomic positions obtained in the diffraction studies and the UWXAFS package[5] to simultaneously fit both polarized XAFS data to the theory by weighting their respective amplitudes as given in Eq. 1 and varying $\theta_{i}$. In addition, adjustable structural parameters were added to the theory, such as changes in half path length, $\delta r_{i}$ and mean square displacements, $\sigma_{i}^{2}$, to account for possible deviations in the local structure from the average structure. Simultaneous fits to both polarizations allow a further reduction in the number of fitting parameters for scattering paths which contribute to both $c$ and $a b$ oriented XAFS. Data and theory in a $k$-range $[3,15] \AA^{-1}$ were weighted by $k^{2}$ and Fourier transformed to $r$-space. The region of $r$-space fitted, including MS, was $r=[1.9,5.3] \AA$. Fig. 1 shows best fits to both orientations for the $x=0.1$ sample at $\mathrm{T}=20 \mathrm{~K}$.

Our main results can be summarized as follows: a) The $\mathrm{Sr}-\mathrm{Cu}$ and $\mathrm{Sr}-\mathrm{La}$ distances and mean square displacements, up to the fifth shell are, within $0.01 \AA$ and $0.001 \AA^{2}$, the same as those as for La-Cu and La-La found in the fittings to La $K$-edge polarized data[6] and in agreement with the distances found by diffraction for the average structure. This result indicates that $\mathrm{Sr}$ indeed substitutes at the La site and is consistent with the $\mathrm{Sr}^{+2}$ and Lat3 ions having nearly the same size. b) Simultaneous fits to SS Sr-O(2) apical and MS Sr-O(2)-Cu paths at $r \approx 4.76 \AA$ show that the O(2) apical oxygen to the $\mathrm{Sr}$ is fully occupied with $\mathrm{N}=0.92 \pm 0.2$. However, it is displaced along the $c$-axis, towards the $\mathrm{Cu}$ atoms, by $\delta r=0.14 \pm 0.1 \AA$ making the $\mathrm{Sr}-\mathrm{O}(2)$ distance $r=2.49 \pm 0.1 \AA$ instead of the $2.35 \AA$ found for $\mathrm{La}-\mathrm{O}(2)$ apical by both XAFS[6] and diffraction studies[7]. The disorder found in the Sr-O(2) apical bond is $\sigma^{2}=0.026 \pm 0.01 \AA^{2}$, 

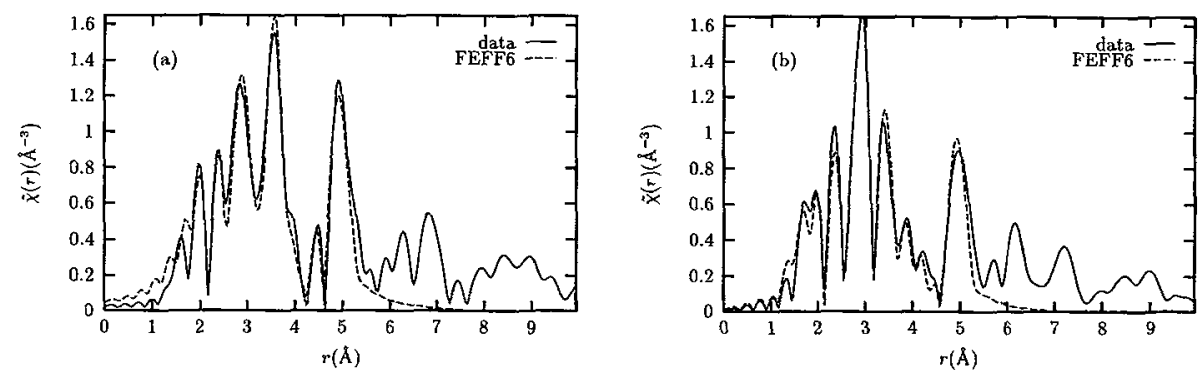

Figure 1: Fits to the $x=0.1$ sample at $\mathrm{T}=20 \mathrm{~K}$ for $\mathrm{X}$-rays polarized (a) along the $c$-axis and (b) in the $a b$-plane. The magnitude of the complex Fourier transform of $k^{2} \chi(k)$ is shown.

an order of magnitude more than for $\mathrm{La}-\mathrm{O}(2)$ apical. The $\delta r$ and $\sigma^{2}$ obtained for the (Sr/La)-O(2)-Cu MS paths, however, are, within $0.01 \AA$ and $0.001 \AA^{2}$, the same for both the $\mathrm{Sr}$ and La central atoms and in agreement with the average structure, indicating that the disorder of the $\mathrm{O}(2)$ apical oxygens is along the c-axis and hence does not affect the path length of the MS paths. The implications of the distortion in the $\mathrm{O}(2)$ apical oxygen distances are many. Since the distortion brings the $\mathrm{O}(2)$ apical much closer to the $\mathrm{Cu}-\mathrm{O}$ planes, it more effectively induces carriers under Sr-doping, and could explain the increase of out-of-plane $02 p_{z}$ character of the doped holes observed by Chen et. al[8]. Also, being closer, the $\mathrm{O}(2)$ apical can serve as a more effective "bridge" between the Cu-O and La-O layers and hence explain the relative increase in out-of-plane conductivity with doping[9]. Finally, adding the $c$-axis static displacement of $0.14 \AA$ to the rms displacement $\left(\sigma^{2}\right)^{1 / 2}=0.16 \AA$ we get an $\mathrm{rms}$ deviation from the average structure $\left\langle u_{z}^{2}\right\rangle^{1 / 2} \simeq 0.3 \AA$. This is remarkably close to the measured rms displacement of the atoms contributing to the diffuse scattering observed by Isaacs $\epsilon t$. al. c) The average structure has two different $\mathrm{La} / \mathrm{Sr}-\mathrm{O}(1)$ distances to the $\mathrm{O}(1)$ oxygens in the $\mathrm{Cu}-\mathrm{O}$ planes at $r_{s}=2.591 \AA$ and $r_{l}=2.675 \AA$. La-O(1) distances were found to be in agreement with the diffraction results $[6]$. The splitting is mainly due to the tilt pattern of the $\mathrm{CuO}_{6}$ octahedra which buckles the Cu-O planes. The Sr XAFS analysis gives for the angles these bonds form with the $c$-axis $\theta^{(s)}=44.1 \pm 6.1^{\circ}$ and $\theta^{(l)}=41.9 \pm 6.2^{\circ}$, respectively, consistent with the values $\theta^{(s, l)}=47.8^{\circ}, 44.2^{\circ}$ found in the diffraction studies[7]. However, the $\mathrm{Sr}-\mathrm{O}(1)$ distances are found to be $r_{s}=2.542(10) \AA$ and $r_{l}=2.734(12) \AA$, respectively. In order to accommodate the changes in $\mathrm{Sr}-\mathrm{O}(1)$ distances while the $\mathrm{Sr}-\mathrm{Cu}$ distances remain unaltered, some $\mathrm{Cu}-\mathrm{O}(1)$ bonds in the basal plane of the $\mathrm{CuO}_{6}$ octahedra must become distorted compared to the average structure. We believe these distortions are not associated with a rigid tilt of the $\mathrm{CuO}_{6}$ octahedra involved because then a corresponding distortion of the $\mathrm{O}(2)$ oxygens forming the apex of the $\mathrm{CuO}_{6}$ octahedra and nearly co-planar with the $\mathrm{Sr}$ should have been observed in our analysis, which was not found.

To summarize, when $\mathrm{Sr}$ substitutes for $\mathrm{La}$ in $\mathrm{La}_{2-x} \mathrm{Sr}_{x} \mathrm{CuO}_{4}$ it resides at the La lattice site but distorts considerably its near neighboring oxygen environment. The $\mathrm{O}(2)$ apical distortion is most likely crucial for the hole doping mechanism in these cuprates as well a for their transport properties. The Sr substitution directly affects the structure of neighboring $\mathrm{CuO}_{6}$ octahedra. This could be an important factor in understanding the mechanism of the Sr-induced structural phase transitions in this material. Finally, the Sr-induced distortions are probably the origin of the observed diffuse scattering of these cuprates.

\section{Acknowledgments}

We are grateful to D. G. Hinks, A. W. Mitchell and J. D. Jorgensen for providing the samples used in this study. The valuable help of F. Perez and M. Suenaga in orienting the samples is greatly appreciated. This work was done under the auspices of DOE Grant No. DE FG06-90ER45425.

\section{References}

[1] Isaacs E.D. et al., Phys. Rev. Lett. 72 (1994) 3421-3424.

[2] Tan Z. et al., Phys. Rev. Lett. 64 (1990) 2715-2718 .

[3] Wu Z.Y. et al, Physica B 208\&209 (1995) 491-492 .

[4] Zabinsky S. et al., Phys. Rev. B 52 (1995) 2995-3009.

[5] Stern E.A. et al., Physica B 208\&209 (1995) 117-120.

[6] Haskel D. et al., Phys. Rev. Lett. 76 (1996) 439-442.

[7] Radaelli P.G. et al., Phys. Rev. B 49 (1994) 4163-4175.

[8] Chen C.T. et al., Phys. Rev. Lett. 68 (1992) 2543-2546.

[9] Kambe S. et. al, Physica C 160 (1989) 35-41. 